\title{
GROSOR DEL FRUTO DE LA ÚLTIMA Y SEGUNDA MANO COMO CRITERIO DE COSECHA EN BANANO ${ }^{1}$
}

\author{
Alfonso Vargas-Calvo ${ }^{2}$
}

\section{RESUMEN}

Grosor del fruto de la última y segunda mano como criterio de cosecha en banano. El objetivo de este trabajo fue determinar la relación entre el grosor del fruto central de las manos última y segunda, como criterio de cosecha en el banano. Las mediciones se efectuaron semanalmente (mm) de la segunda a la décima semana después de la floración y fueron transformadas a treintaidosavos de pulgada (tdp) donde $1 \mathrm{tdp}=0,7914 \mathrm{~mm}$, para la generación de ecuaciones de regresión para ambas manos, considerando, o ignorando, el tamaño del racimo. El grosor del fruto central de la última mano fue estimado a partir del grosor del fruto central de la segunda mano, y viceversa para el grosor del fruto de la segunda mano. Los valores de grosor del fruto central de la última mano (valores $\mathrm{Y}$ ) obtenidos aplicando los grosores mínimos preestablecidos de cosecha para el fruto central de la segunda mano (valores $\mathrm{x}$ : 43; 44 y $45 \mathrm{tdp}$ ) fueron superiores a 40 tdp, independientemente del tamaño del racimo, e inclusive superó en la mayoría de las veces, a 41 tdp. Inversamente, aplicando los valores mínimos de grosor de la última mano, predefinidos para la cosecha (valores x: 39; 40 y 41 tdp), se obtuvieron valores de grosor del fruto central de la segunda mano (valores Y), que no superaron el valor de 43 tdp excepto para $\mathrm{x}=41$ en los racimos de siete a nueve manos. Los racimos cosechados con la exigencia mínima de empaque aplicada para la última mano, tuvieron menos peso, por estar conformados de frutos con menor grosor, que aquellos que se cosecharían mediante la medición en la segunda mano.

Palabras clave: Crecimiento del fruto, criterio preestablecido de cosecha, Grande Naine, medición del fruto, Musa AAA.

\begin{abstract}
Thickness of the last and second "fruit hands" as harvest criteria in banana. The objective of this work was to determine the relationship between the thickness of the last and second hand of fruits as harvest criteria in banana. The measurements were done weekly $(\mathrm{mm})$ from the second to the tenth weeks after flowering, and were transformed to thirty two inch/22 (tti), where $1 \mathrm{tti}=0,794 \mathrm{~mm}$ for the calculation of regression equations for both fruit hands, considering or ignoring the bunch size. The thickness of the central fruit of the last hand was estimated from the central thickness of the second hand, and vice versa for the fruit thickness of the second hand. The values of thickness of the central fruit of the last hand (Y values), obtained by applying a pre-established minimum value for the harvest of the second hand central fruit (x values: 43, 44 and $45 \mathrm{tti}$ ) were over $40 \mathrm{tti}$, regardless of the bunch size, and even, exceeded in most cases, $41 \mathrm{tti}$. Conversely, applying the minimum values of the central fruit of the last hand predefined for harvest ( $\mathrm{x}$ values: 39,40 and $41 \mathrm{tti}$ ), were obtained thickness values of the central fruit of the second hand ( $Y$ values) that did not exceed the value of $43 \mathrm{tti}$, except for $\mathrm{x}=41 \mathrm{tti}$ in bunches of 7 to 9 hands. The results clearly demonstrate that bunches harvested with the minimum requirement of packing in the last hand, would have less weight because they are formed by fruits of less thickness than those reaped by measuring the second hand.
\end{abstract}

Key words: Fruit growth, fruit measurement, Grande Naine, Musa AAA, pre-established harvest criteria.

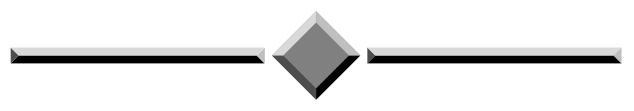

1 Recibido: 7 de octubre, 2011. Aceptado: 12 de marzo, 2012.

2 Corporación Bananera Nacional (CORBANA, S.A.). Limón, Costa Rica. Apdo. postal 506-2763-3055. alfvarga@corbana.co.cr 


\section{INTRODUCCIÓN}

La cosecha es probablemente la operación más delicada de la producción de banano, ya que bajo las estrategias actuales, se debe cosechar el racimo de acuerdo con la edad y el grosor requerido, así como el correcto manejo del fruto durante el transporte y empaque (Robinson 1996, United Brands 1975). El grosor del fruto, en conjunto con el intervalo floracióncosecha (edad), es la variable que mejor define el momento apropiado de cosecha del racimo, puesto que, independientemente de la región de producción o del número de manos, éstos son los factores limitantes y dominantes para la comercialización orientada a la exportación (Jaramillo 1982).

El grosor adecuado de cosecha varía de acuerdo a la distancia entre el sitio de producción y el mercado específico de destino, relación oferta demanda, tipo de cultivar, estado fisiológico de la plantación, clima o enfermedades y plagas (Soto 1992). El anterior autor define el grosor óptimo como aquel estado de madurez fisiológica del fruto que permite el máximo aprovechamiento del racimo, sin que exista riesgo de maduración durante el transporte, manteniendo la lozanía y calidad propias de una fruta para consumo fresco.

Generalmente la cosecha de los racimos de banano para exportación se realiza cuando éstos cuando aún penden de la planta, y depende del grosor del fruto central de la fila externa de la segunda mano. No obstante en ocasiones, dicha medición se realiza en el fruto central de la última mano, donde el criterio de cosecha generalmente está dado en función del grosor mínimo exigido para empaque.

Las diferencias en el grosor del fruto en racimos de diferente tamaño son difíciles de notar hasta la quinta mano. A partir de allí, dada una misma posición de la mano en el racimo, el grosor del fruto de las manos siguientes y de la última en especial, disminuye conforme aumenta el tamaño del racimo (Vargas y Blanco 2004). También Robinson (1966) menciona que existió variación del tamaño de los frutos en el racimo, donde aquellos ubicados en la parte inferior son de 30 a $40 \%$ más pequeños que los presentes en la parte superior, gradiente que de acuerdo con dicho autor, contribuye a la variabilidad en el rendimiento en racimos de banano cosechados a la misma edad. Esta gradiente en las dimensiones de los frutos, sería la consecuencia (Jullien et al. 2001) del inicio y la finalización más tardía de la división celular, en aquellos frutos de las manos inferiores con respecto a los de las manos superiores, con una mayor cantidad de células en estas últimas al final de dicha fase. En concordancia, dichos autores concluyen que la variación en el peso del fruto que se manifiesta dentro del racimo está relacionada con la edad y el número de células.

Cuando el grosor del fruto se determina con respecto al tamaño del racimo, aquellos de mayor tamaño presentan, a una misma edad, mayor grosor que los de menor tamaño, y Soto y Ruiz (1992) sugieren que por lo tanto, sería lógico efectuar la determinación del grosor de cosecha en la última mano, en lugar de la tradicionalmente usada segunda mano. No obstante, Jaramillo (1982) indica que la diferencia entre el grosor de los frutos de la segunda y última mano (fila externa) en los racimos de tamaño pequeño es de 3,0 a 4,0 treintaidosavos de pulgada $\mathrm{y}$, en los de tamaño mediano y grande, de 5,0 a 6,5 treintaidosavos de pulgada, dicha diferencia debe ser considerada. Ello debido básicamente a la necesidad de mezclar adecuadamente grupos de gajos con frutos de distinto grosor dentro de la caja, como parte determinante en la cosecha, según sea la distancia al mercado de destino. De acuerdo con dicho autor, el grosor de corta para la segunda mano y establecido de antemano por la finca debería ser tal, que permita a la última mano alcanzar el grosor requerido de empaque. Si esto no sucede, además de la pérdida de peso del racimo, es probable, que ocurra también, la pérdida de manos inferiores.

La información disponible sobre el tema es limitada y no existen documentos que indiquen y cuantifiquen la asociación entre el grosor del fruto de la última y segunda mano durante su desarrollo en el racimo y particularmente con respecto a las etapas de cosecha. Su conocimiento sería de gran ayuda para una mejor determinación y aplicación del criterio de cosecha respectivo, particularmente cuando esta se realice en función de la última mano. Por ello, el objetivo de este estudio consistió en determinar la relación entre el grosor del fruto central de la fila externa de las manos última y segunda durante el desarrollo del racimo de banano (Musa AAA, cv. Grande Naine) y las implicaciones productivas del uso de una u otra como criterio de cosecha. 


\section{MATERIALES Y MÉTODOS}

Las mediciones al racimo se efectuaron de enero a mayo del 2007 en una sección de cultivo de primera generación perteneciente a una finca comercial localizada en la provincia de Heredia, cantón de Sarapiquí con influencia del Caribe de Costa Rica. La finca fue sembrada en 1993 con el cv. Grande Naine y el área experimental fue renovada y conformada en domos en el año 2006 con el mismo cultivar a una densidad de población de 1666 plantas/ha. El suelo presentó un pH de 4,9 y contenidos de $\mathrm{Ca}, \mathrm{Mg}$ y K de 8,65; 4,4 y $0,35 \mathrm{cmol}(+) / 1$, respectivamente y un $2,8 \%$ de materia orgánica. Estuvo constituido por porcentajes de arena, arcilla y limo de 20,50 y $30 \%$, respectivamente, y clasificado texturalmente como Arcilloso moderado. Durante el período experimental la precipitación total fue de $1013 \mathrm{~mm}$, la temperatura promedio máxima y mínima de 30,9 y $21,3^{\circ} \mathrm{C}$ y la humedad promedio de $85,01 \%$.

La fertilización anual durante el año 2006, período en que el área experimental fue sembrada, se realizó con $224 \mathrm{~kg}$ de $\mathrm{N}, 3 \mathrm{~kg}$ de $\mathrm{P}_{2} \mathrm{O}_{5}, 351 \mathrm{~kg}$ de $\mathrm{K}_{2} \mathrm{O}$, $65 \mathrm{~kg}$ de $\mathrm{MgO}, 453 \mathrm{~kg}$ de $\mathrm{CaO}, 68 \mathrm{~kg}$ de $\mathrm{S}, 7 \mathrm{~kg}$ de $\mathrm{Zn}$ y $3 \mathrm{~kg}$ de $\mathrm{B}$ divididos en 8 ciclos, excepto el $\mathrm{CaO}$ que se realizó en uno.

El combate de la Sigatoka Negra (Mycosphaerella fijiensis) se realizó mediante la aspersión aérea de fungicidas sistémicos y protectores. Los mismos se aplicaron rotativamente y en mezcla con aceite agrícola (5-10 1/ha) en un volumen total de aplicación de 18 a 25 l/ha, de acuerdo con los requerimientos y estrategias de combate propias de la finca y época climática. Operaciones semanales de deshoja sanitaria se utilizaron como complemento al combate químico.

El embolse de los racimos se realizó una semana, y el desmane, dos semanas después de la floración, ésta ultima labor acompañada por la eliminación de la mano falsa y generalmente de la chira. Se consideró como mano falsa al conjunto de flores en donde una o más se desarrollaron anormalmente, permaneciendo como una reminiscencia del ovario. Como mano verdadera se consideró aquel grupo de flores cuyos gineceos se desarrollaron normalmente. El desmane consistió en la remoción de dos manos en los racimos de siete, ocho y nueve y de tres manos en los de diez y once manos verdaderas a la floración. No se realizó el desdede lateral de frutos ni la desflora.
Las mediciones de grosor del fruto central de la fila externa de la última y segunda mano se efectuaron semanalmente a partir de la segunda semana después de la floración y en forma sucesiva durante las diez semanas posteriores conforme las plantas del área experimental fueron floreciendo. Por tal motivo la cantidad de racimos usados correspondió a la distribución por tamaño que en esa sección experimental se expresó. La labor se efectuó en la parte media del fruto respectivo, perpendicularmente al plano de la curvatura, con la ayuda de un calibrador tipo Vernier graduado en mm. Los valores obtenidos fueron convertidos a treintaidosavos de pulgada (tdp) en donde $1 \mathrm{tdp}=0,794 \mathrm{~mm}$. La cosecha de los racimos se realizó once semanas después del embolse. Se consideraron para efectos del experimento tres diferentes grosores preestablecidos de cosecha, definidos para el fruto central de la segunda ( $43 ; 44$ y 45 tdp) y última ( $39 ; 40$ y 41 tdp) mano.

El conjunto de datos se analizó mediante regresión. Se generaron ecuaciones con las que se determinó la tasa de engrosamiento semanal considerando e ignorando el tamaño de racimo para el fruto central de la segunda y última mano. Asimismo se estimó, mediante la inclusión de los grosores preestablecidos de cosecha en las ecuaciones, el grosor del fruto central de una de dichas manos a partir de la medición del fruto central de la otra, considerando e ignorando el tamaño de racimo.

\section{RESULTADOS Y DISCUSIÓN}

Las pendientes de las ecuaciones estimadas del Cuadro 1 representan tasas semanales de engrosamiento del fruto central de las manos segunda y última, las cuales variaron según el tamaño de racimo, de 2,18 tdp (cinco manos) a 2,25 tdp (nueve manos) en la segunda mano y de 2,17 tdp (cinco manos) a 2,03 tdp ( 9 manos) en la última mano. El engrosamiento semanal promedio (Figura 1), obtenido ignorando el tamaño del racimo fue de 2,21 tdp para la segunda mano y de 2,07 tdp para la última.

La mayor tasa de engrosamiento que presentó el fruto de la segunda mano con respecto al de la última, es congruente con los resultados de Jullien et al. (2001) quienes indican que ello tiene su origen en una finalización más temprana de la división celular del fruto en la mano superior que involucra además una mayor cantidad de células al término de dicho período. 
Cuadro 1. Ecuaciones de regresión lineal del grosor $(\mathrm{Y})$ del fruto central de la segunda y última mano, de racimos de banano (Musa AAA, cv. Grande Naine), en función de la edad del racimo (x), para diferente número de manos presentes a la cosecha (unidades: $Y$ en treintaidosavos de pulgada ${ }^{1}$ (tdp), $x$ en semanas). Sarapiquí, Heredia, Costa Rica. 2007.

\begin{tabular}{|c|c|c|c|c|c|}
\hline \multirow{2}{*}{$\begin{array}{l}\text { Manos } \\
\text { presentes }\end{array}$} & \multirow[t]{2}{*}{$\mathbf{n}$} & \multicolumn{2}{|c|}{ Segunda mano } & \multicolumn{2}{|c|}{ Última mano } \\
\hline & & Ecuación & $\mathbf{R}^{2}$ & Ecuación & $\mathbf{R}^{2}$ \\
\hline 5 & 49 & $Y=2,183 x+22,133$ & 0,93 & $Y=2,171 x+20,724$ & 0,94 \\
\hline 6 & 131 & $Y=2,191 x+21,899$ & 0,93 & $Y=2,110 x+20,801$ & 0,94 \\
\hline 7 & 241 & $Y=2,220 x+21,383$ & 0,93 & $Y=2,054 x+20,703$ & 0,94 \\
\hline 8 & 93 & $Y=2,202 x+21,254$ & 0,93 & $Y=2,055 x+20,348$ & 0,93 \\
\hline 9 & 10 & $Y=2,250 x+21,516$ & 0,95 & $Y=2,030 x+20,645$ & 0,94 \\
\hline
\end{tabular}

${ }^{1}$ Un treintaidosavo de pulgada $=0,794 \mathrm{~mm}$

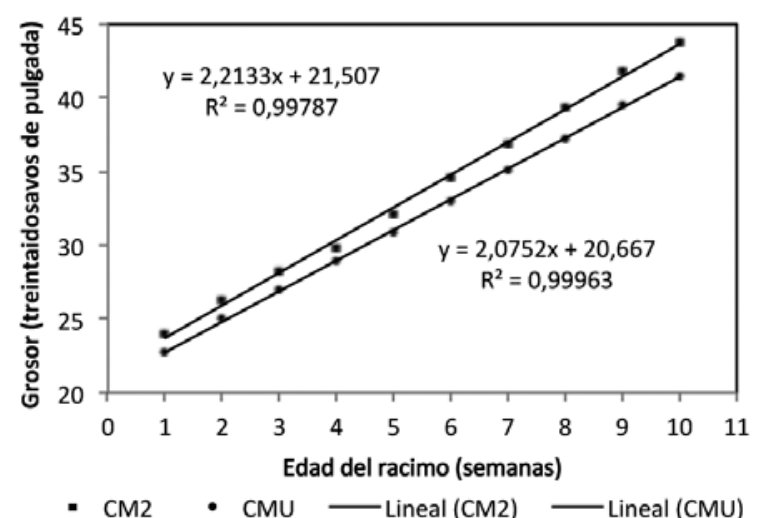

Figura 1. Efecto lineal promedio de la edad del racimo en el grosor del fruto central de la segunda y última mano de racimos de banano (Musa AAA, cv. Grande Naine) ignorando el tamaño del racimo (manos presentes y número de racimos considerados según indica el Cuadro 1). CM2: grosor de la mano 2, CMU: grosor de la mano última, ambos en treintaidosavos de pulgada (un treintaidosavo de pulgada $=0,794 \mathrm{~mm})$. Sarapiquí, Heredia, Costa Rica. 2007.

El grosor del fruto central de la última mano fue estimado a partir del grosor del fruto central de la segunda mano; asimismo, en forma inversa, éste fue estimado a partir de aquel. Las ecuaciones obtenidas se presentan en los Cuadros 2 y 3.

Los valores de grosor del fruto central de la última mano (valores Y) obtenidos aplicando los grosores mínimos preestablecidos de cosecha para el fruto central de la segunda mano (valores x: 43; 44 y 45 tdp) fueron superiores a 40 tdp (Cuadro 2) independientemente del tamaño del racimo, e inclusive superó en la mayoría de las veces, a 41 tdp, que define la exigencia máxima de grosor de empaque en la actualidad.

Inversamente, aplicando los valores mínimos de grosor de la última mano, predefinidos para la cosecha (valores x: 39; 40 y 41 tdp), se obtuvieron valores de grosor del fruto central de la segunda mano (valores Y), que no superaron el valor de 43 tdp (Cuadro 3), excepto para $\mathrm{x}=41$ en los racimos de siete a nueve manos. El grosor de 43 tdp en la segunda mano representa el mínimo usado para la cosecha del racimo

Dado lo anterior, para cosechar racimos con grosores superiores a $43 \mathrm{tdp}$ en el fruto de la segunda mano y con el grosor requerido de la última mano como criterio de cosecha, el grosor a medir en dicha mano debería ser, de acuerdo con las estimaciones generadas en este estudio, superiores a $41 \mathrm{tdp}$. Grosores inferiores supondrían una pérdida de peso toda vez que de acuerdo con Soto y Ruiz (1992) cada tdp representa $1,72 \mathrm{~kg}$.

Independientemente de la medida preestablecida a cosecha de cada mano, el grosor estimado de la última mano disminuyó y por el contrario, el grosor estimado de la segunda se incrementó, conforme aumentó el tamaño del racimo. La diferencia en grosor entre los frutos centrales de ambas manos también aumentó con el desarrollo del racimo. De manera general e ignorando tanto el tamaño del racimo como la medida preestablecida de cosecha, dicha diferencia en grosor estuvo comprendida entre 2,0 y 2,4 tdp. 
Cuadro 2. Valores predichos (treintaidosavos de pulgada ${ }^{1}(\mathrm{tdp})$ ) de grosor (Y) de la última mano de banano (Musa AAA cv Grande Naine), aplicando valores preestablecidos de grosor de la mano 2 (x) a ecuaciones estimadas previamente, en racimos de cinco a nueve manos. Sarapiquí, Heredia, Costa Rica.2007.

\begin{tabular}{lcccccc}
\hline \multicolumn{7}{c}{ Número de manos por racimo presentes a la cosecha } \\
$\mathbf{x}=\mathbf{G M 2}^{2}$ & $\mathbf{5}$ & $\mathbf{6}$ & $\mathbf{7}$ & $\mathbf{8}$ & $\mathbf{9}$ & Promedio $^{3}$ \\
\cline { 2 - 5 } & $\mathrm{Y}=-0,674+$ & $\mathrm{Y}=0,238+$ & $\mathrm{Y}=1,408+$ & $\mathrm{Y}=0,954+$ & $\mathrm{Y}=1,580+$ & $\mathrm{Y}=0,900+$ \\
& $0,976 \mathrm{x}$ & $0,947 \mathrm{x}$ & $0,910 \mathrm{x}$ & $0,920 \mathrm{x}$ & $0,891 \mathrm{x}$ & $0,925 \mathrm{x}$ \\
43 & $\mathrm{R}^{2}=0,98$ & $\mathrm{R}^{2}=0,97$ & $\mathrm{R}^{2}=0,97$ & $\mathrm{R}^{2}=0,97$ & $\mathrm{R}^{2}=0,97$ & $\mathrm{R}^{2}=0,97$ \\
44 & 41,3 & 41,0 & 40,5 & 40,5 & 39,9 & 40,7 \\
45 & 42,3 & 41,9 & 41,4 & 41,4 & 40,8 & 41,6 \\
$\mathrm{n}^{4}$ & 43,2 & 42,9 & 42,4 & 42,3 & 41,7 & 42,5 \\
\hline
\end{tabular}

${ }^{1}$ Un treintaidosavo de pulgada $=0,794 \mathrm{~mm},{ }^{2}$ Grosor de la mano, ${ }^{3}$ Ignora el tamaño de racimo a la cosecha. ${ }^{4}$ Número de racimos evaluados.

Cuadro 3. Valores predichos (treintaidosavos de pulgada ${ }^{1}(\mathrm{tdp})$ ) de grosor (Y) de la segunda mano de banano (Musa AAA cv Grande Naine), aplicando valores preestablecidos de grosor de la última mano (x) a ecuaciones estimadas previamente, en racimos de cinco a nueve manos. Sarapiquí, Heredia, Costa Rica. 2007.

\begin{tabular}{|c|c|c|c|c|c|c|}
\hline \multirow[b]{2}{*}{$\mathbf{x}=\mathbf{G M U}^{2}$} & \multicolumn{5}{|c|}{ Número de manos por racimo presentes a la cosecha } & \multirow[b]{2}{*}{ Promedio $^{3}$} \\
\hline & 5 & 6 & 7 & 8 & 9 & \\
\hline & $\begin{array}{c}\mathrm{Y}=1,486+ \\
0,999 \mathrm{x} \\
\mathrm{R}^{2}=0,98\end{array}$ & $\begin{array}{c}\mathrm{Y}=0,710+ \\
1,026 \mathrm{x} \\
\mathrm{R}^{2}=0,97\end{array}$ & $\begin{array}{c}Y=-0,621+ \\
1,069 x \\
R^{2}=0,97\end{array}$ & $\begin{array}{c}Y=-0,047+ \\
1,056 \mathrm{x} \\
\mathrm{R}^{2}=0,97\end{array}$ & $\begin{array}{c}Y=-0,697+ \\
1,087 x \\
R^{2}=0,97\end{array}$ & $\begin{array}{c}-0,009+1,050 x \\
\mathrm{R}^{2}=0,97\end{array}$ \\
\hline 39 & 40,4 & 40,7 & 41,1 & 41,1 & 41,7 & 40,9 \\
\hline 40 & 41,4 & 41,8 & 42,1 & 42,2 & 42,8 & 42,0 \\
\hline 41 & 42,4 & 42,8 & 43,2 & 43,2 & 43,9 & 43,0 \\
\hline $\mathrm{n}^{4}$ & 49 & 131 & 241 & 93 & 10 & 524 \\
\hline
\end{tabular}

${ }^{1}$ Un treintaidosavo de pulgada $=0,794 \mathrm{~mm},{ }^{2}$ Grosor de la mano, ${ }^{3}$ Ignora el tamaño de racimo a la cosecha. ${ }^{4}$ Número de racimos evaluados.

La disminución del grosor de la última mano, y el aumento de dicha variable en la segunda conforme se incrementó el tamaño del racimo, es congruente con lo indicado por Vargas y Blanco (2004), Jullien et al. (2001), Robinson (1996), Soto y Ruiz (1992) y Jaramillo (1982). Este comportamiento está dado por una gradiente decreciente en las dimensiones del fruto de las manos superiores a las inferiores que ocurre por lo general luego de la quinta mano (Vargas y Blanco 2004) en los bananos del subgrupo Cavendish usados para la exportación.
Esta particularidad en el patrón de crecimiento de los frutos puede también apreciarse en las diferencias en grosor entre la segunda y la última mano, las cuales aumentaron en magnitud conforme el tamaño de racimo fue mayor, condición que también la señaló Jaramillo (1972).

Las ecuaciones de regresión generadas en este estudio, con el sustento de su alto coeficiente de determinación, podrían estimar con una mejor precisión el grosor del fruto de la última o segunda mano a través de su desarrollo en el tiempo y en especial durante la 
etapa de cosecha. Ello considerando el tamaño de racimo $\left(\mathrm{R}^{2}\right.$ de 0,93 a 0,98$)$ o independientemente del mismo $\left(\mathrm{R}^{2}\right.$ de 0,99$)$ mediante la sustitución en la ecuación correspondiente el grosor medido o predeterminado de una de las dos manos antes mencionadas.

Bajo las estrategias actuales de cosecha (edad del racimo y grosor preestablecido del fruto central), estos resultados demuestran claramente que los racimos que se cosechan en función de la exigencia mínima de empaque aplicada para la última mano tendrían menos peso que aquellos que se cosecharían mediante el uso de la segunda mano como criterio de cosecha.

De ese modo, la consideración de la última mano y de su fruto central como indicadores de cosecha, no sería, en contraste con lo indicado por Soto y Ruiz (1992) un criterio apropiado para definir la cosecha del racimo bajo condiciones normales de plantación y de clima. De tal manera, el criterio de cosecha basado en la medición del grosor de la segunda mano sería el más apropiado, toda vez que su aplicación, garantiza la cosecha de frutos con mejores grosores, en especial de aquellos provenientes de manos superiores. Esto es asimismo vinculante cuando se deban realizar cosechas más tempranas ('precorta') ocasionadas por defoliaciones severas provocadas por la Sigatoka negra (Mycosphaerella fijiensis) o cuando existan problemas en el engrosamiento de los frutos (clima y/o nutrición), situación que obliga en ocasiones a los productores a realizar la medición de cosecha en la última mano, referida por lo general con base a la exigencia mínima de empaque.

La aplicación general de este concepto podría ser de gran ayuda para determinar, bajo las condiciones particulares de cada finca, el criterio de cosecha en la mano a medir que brinde un mejor aprovechamiento del racimo, especialmente en aquellos casos en que dicho criterio estuviese dado por la medición del fruto central de la última mano.

\section{LITERATURA CITADA}

Jaramillo, R. 1982. Las principales características morfológicas del fruto de banano, variedad Cavendish Gigante (Musa AAA) en Costa Rica. Unión de Países Exportadores de Banano. Panamá. 42 p.

Jullien, A; Malezieux, E; Michaux-Ferrieres, N; Chillet, M; Ney, B. 2001. Within-bunch variability in banana fruit weight: importance of development lag between fruits. Annals of Botany 87:101-108.

Robinson, JC. 1996. Bananas and plantains. Institute for Tropical and Subtropical Crops, South Africa. CAB International. Wallingford, UK. 238 p.

Soto, M. 1992. Cosecha y empaque de la fruta. In Soto, M. ed. Banano Cultivo y Comercialización. 2 ed. Litografía e Imprenta LIL, S.A. San José, CR. 649 p.

Soto, M; Ruiz, E. 1992. Descripción botánica. In Soto, M. ed. Bananos. Cultivo y Comercialización. 2 ed. Litografía e Imprenta LIL, S.A. San José, CR. 649 p.

Vargas, A; Blanco, F. 2004. Metodología para estimar la intensidad de desmane en racimos de banano (Musa AAA, cv. Valery). CORBANA 30(57):107-119.

United Brands. 1975. Guía práctica para el cultivo del banano. Departamento de Investigaciones Agrícolas Tropicales. La Lima, Honduras. 224 p. 